\title{
A REVIEW: MYSTERIOUS CASE OF A QUBIT's STATE
}

\author{
Subhasish Sarkar \\ Senior SQA Engineer \\ BMC Software India Pvt. Ltd, Pune, Maharashtra, India
}

\begin{abstract}
This is the author's second 'Review Paper' on the fascinating, exciting and challenging world of "Quantum Computing". While, in the first paper, the author took the reader, supposedly having no knowledge of Quantum Mechanics and Quantum Computing, on a "Beginner's" journey into the interesting world of Quantum Physics and Quantum Computing, this review paper tries to dig deep into the curious world of the Qubit, its states and all the associated mysteries surrounding a Qubit and its 'simultaneous' many states.
\end{abstract}

Keywords - Quantum Computing, Qubit, Quantum Bit, Quantum Computer, Superposition

\section{INTRODUCTION}

For the "classical" computers that we know of, use or interact with in some form or shape, every day or pretty much thereof, the 'Bit' is the fundamental unit of storing, retrieving and processing information. Just as the "classical" (conventional) computers are made up of Bits, Quantum Computers are made up of what are called Quantum Bits, or simply, Qubits.

A 'classical Bit' can be thought of having a state which is either 0 or 1 . Similarly, a Qubit also has got a state.

The author's discussion, in this "review paper", precisely revolves around a Qubit and its state. Readers willing to pursue an in-depth study and understanding of the other concepts of the field of Quantum Computing should immensely benefit by referring to some of or all the materials [1 - 15] specified in the 'REFERENCE' section below.

\section{SYSTEM AND METHOD}

\section{A. State Space -}

While the state of a 'classical bit' is a number, either 0 or 1 , a qubit's state is a vector.

In fact, a qubit's state is a vector in a two-dimensional vector space, which is known as the state space. ----- $\mathbf{i}$

\section{B. Computational Basis States -}

Just like the 'classical bit', a qubit also has states (call those "Quantum States" rather than merely "states" for a qubit) that correspond to a classical bit's 0 and 1 states. In fact, it is better to say that these two states are special quantum states of a qubit and are called computational basis states.

A qubit's quantum state that corresponds to the classical bit state of 0 is usually denoted by $|\mathbf{0}\rangle$. Similarly, a qubit's quantum state that corresponds to the classical bit state of 1 is usually denoted by $|1\rangle$.

These state notations using | and $\rangle$ are referred to as $\boldsymbol{k e t}$ notations, and $|\mathbf{0}\rangle$ and $|1\rangle$ are called kets. The symbols | and \ usually have no special meanings, except for the fact that those are used to symbolize quantum states.

A ket is simply a vector. Therefore, in terms of vector notations, kets $|\mathbf{0}\rangle$ and $|1\rangle$ are represented as follows.

$$
\begin{aligned}
& |0\rangle=\left[\begin{array}{l}
1 \\
0
\end{array}\right] \\
& |1\rangle=\left[\begin{array}{l}
0 \\
1
\end{array}\right]
\end{aligned}
$$

NOTE: -

Although a ket is a vector, a ket is used to denote quantum state notations like $|\mathbf{0}\rangle$ or $|1\rangle$.

We do not usually refer to a normal vector notation like

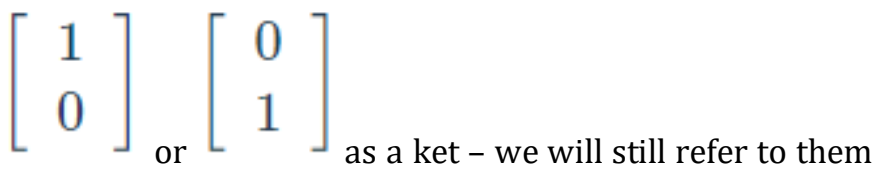
as a 'vector'.

\section{Concept of Superposition -}

Thus far, we have come to know that a qubit can have two possible quantum states of $|\mathbf{0}\rangle$ and $|1\rangle$, which are called computational basis states. However, qubits can exist in many 
more possible states - remember that a qubit's quantum state is a two-dimensional vector in the state space. ----- from $\mathbf{i}$ above

Let us consider the following diagram, for example.

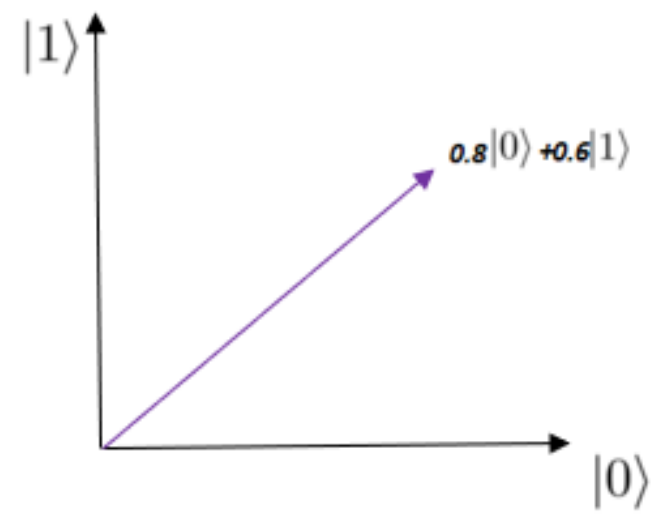

Fig. 1. Diagrammatical illustration of the vector nature of a qubit's quantum state in the two-dimensional vector state space

The figure represents that the qubit's quantum state " $0.8|\mathbf{0}\rangle+0.6|1\rangle$ " is 0.8 times the $|\mathbf{0}\rangle$ vector plus 0.6 times the vector $|1\rangle$.

$$
\begin{aligned}
& 0.8|0\rangle+0.6|1\rangle=0.8\left[\begin{array}{l}
1 \\
0
\end{array}\right]+0.6\left[\begin{array}{l}
0 \\
1
\end{array}\right] \\
& =\left[\begin{array}{c}
0.8 \\
0
\end{array}\right]+\left[\begin{array}{c}
0 \\
0.6
\end{array}\right]=\left[\begin{array}{l}
0.8 \\
0.6
\end{array}\right]
\end{aligned}
$$

At this point, it would probably be good to introduce the fact that a qubit's quantum state is a two-dimensional complex vector in the state space. In other words, the vector can have complex numbers as entries. However, do take note of the fact that the immediately previous vector calculation had involved only real number entries. Similarly, the vector notations of the computational basis states also involve real number entries only.

However, a general quantum state does involve complex number entries. For example, let us consider the following quantum state vector.

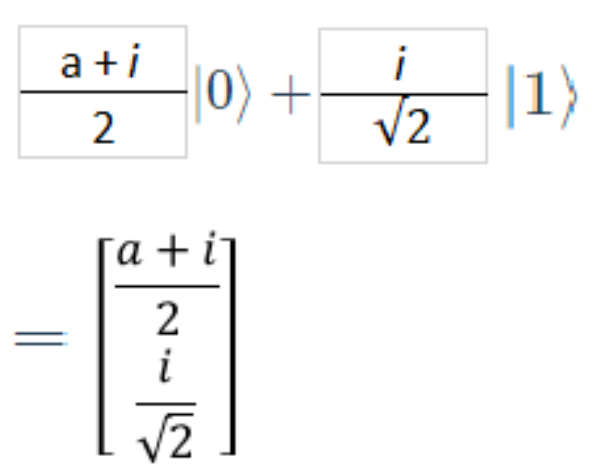

Therefore, we can now say that a qubit's quantum state is a two-dimensional vector in a complex vector state space.

And, now, the time is ripe to get introduced to the concept of Superposition. If we again consider the qubit's quantum state " $0.8|\mathbf{0}\rangle+0.6|1\rangle$ ", we can technically say that the quantum state is a superposition of the computational basis states $|\mathbf{0}\rangle$ and $|1\rangle$. Technically speaking, we can also say that the qubit's quantum state " $0.8|\mathbf{0}\rangle+0.6|1\rangle$ " is a linear combination of the computational basis states $|\mathbf{0}\rangle$ and $|1\rangle$.

\section{Amplitude -}

An amplitude is the coefficient of a qubit's superposition quantum state. If we consider the qubit's superposition quantum state " $0.8|\mathbf{0}\rangle+0.6|1\rangle$ ", we can say that the amplitude for $|\mathbf{0}\rangle$ is 0.8 , and the amplitude for $|\mathbf{1}\rangle$ is 0.6 .

It is worth noting that the sum of the squares of the amplitudes must be equal to 1 . Thus, for the qubit's superposition quantum state " $0.8|\mathbf{0}\rangle+0.6|1\rangle$ ”, $(0.8)^{2}+(0.6)^{2}$ $=0.64+0.36=1$.

If we are to generalize this, let us assume that $\alpha$ and $\beta$ be the amplitudes of a qubit's superposition quantum state. We must remember here that the amplitudes can be complex numbers. So, the qubit's superposition quantum state becomes $\alpha|\mathbf{0}\rangle+\beta|1\rangle$, such that $|\alpha|^{2}+|\beta|^{2}=1$, and this is what is known as the Normalization Constraint.

\section{E. Normalization Constraint -}

Let us consider the following diagram. 


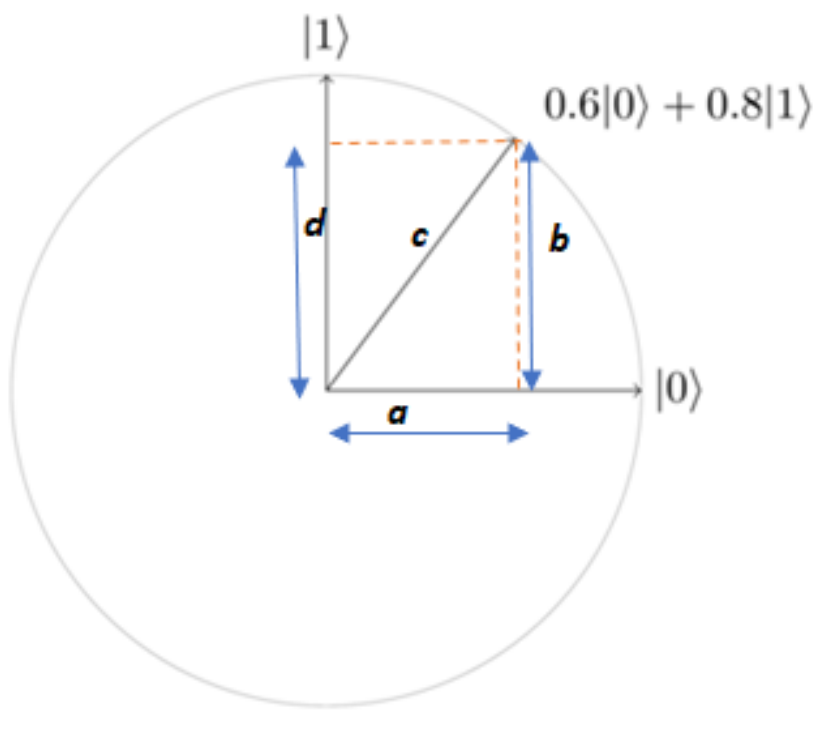

Fig. 2. Diagrammatical illustration of a qubit's computational basis states and superposition quantum state

From the above figure, it can be inferred that $d=0.8$. And, $b=$ $\mathrm{d}=0.8 . \mathrm{a}=0.6$.

Using the Pythagorean theorem, also known as Pythagoras' theorem, $a^{2}+b^{2}=c^{2}$

$$
\begin{aligned}
& \Rightarrow c^{2}=(0.6)^{2}+(0.8)^{2}-----\mathbf{i i} \\
& \Rightarrow c^{2}=0.36+0.64 \\
& \Rightarrow c^{2}=1 \text {---- iii } \\
& \Rightarrow c=\sqrt{1} \\
& \Rightarrow c=1----\mathbf{i v}
\end{aligned}
$$

In other words, the radius of the circle is 1 . Therefore, you can think of the lengths of a qubit's computational basis states $|\mathbf{0}\rangle$ and $|1\rangle$ as being $1 .|\mathbf{0}\rangle$ and $|1\rangle$ are, therefore, orthonormal vectors.

A vector having a unit length (length of one) is said to be a normal vector. $|\mathbf{0}\rangle$ and $|1\rangle$ are, therefore, normal vectors because each has a unit length.

$>$ If two vectors are at right angles to each other, they are said to be orthogonal. Looking at Figure 2, it is evident that $|\mathbf{0}\rangle$ and $|1\rangle$ are orthogonal vectors.

$>$ The dot product of two orthogonal vectors is zero.

$$
|0\rangle \cdot|1\rangle=\left[\begin{array}{l}
1 \\
0
\end{array}\right] \cdot\left[\begin{array}{l}
0 \\
1
\end{array}\right]
$$

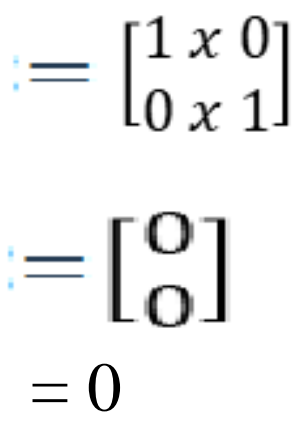

$>$ Two normal, orthogonal vectors are said to be orthonormal. It can, therefore, be concluded that $|\mathbf{0}\rangle$ and $|1\rangle$ are orthonormal vectors.

Also, from ii, iii and iv, it can be inferred that the normalization constraint means that a qubit's superposition quantum state has a unit length; in other words, a qubit's superposition quantum state is a unit or normalized vector and that is also a reason why the constraint $|\alpha|^{2}+|\beta|^{2}=1$ is called the normalization constraint.

Now, taking into consideration all the ideas till this point, we can say that a qubit's quantum state is a unit length vector in a two-dimensional complex vector space that is known as the state space.

\section{NOTE: -}

A qubit's computational basis state $|\mathbf{0}\rangle$ should not be misunderstood as the zero vector, often denoted by 0 in the vector space. If you look at Figure 2, the zero vector is at the exact center point of the circle.

$0=\left[\begin{array}{l}0 \\ 0\end{array}\right]$

However, the qubit's computational basis state $|\mathbf{0}\rangle$ vector is something that is fundamentally different from the zero vector.

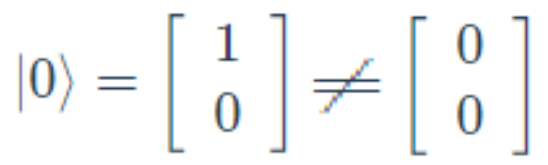

Therefore, $|0\rangle \neq 0$ 


\section{International Journal of Engineering Applied Sciences and Technology, 2020 Vol. 5, Issue 1, ISSN No. 2455-2143, Pages 395-398 \\ Published Online May 2020 in IJEAST (http://www.ijeast.com)}

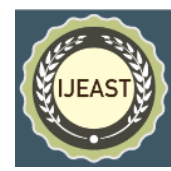

\section{CONCLUSION}

So, to conclude, I would like to mention that the most common description of a qubit's superposition quantum state is probably that the qubit is simultaneously in the computational basis states $|\mathbf{0}\rangle$ and $|\mathbf{1}\rangle$ when it is in some superposition quantum state.

\section{ACKNOWLEDGEMENT}

The author sincerely bows down in all true respect, love and devotion before the ALMIGHTY GOD without whose grace and mercy, nothing is possible in this materialistic world.

The author wishes to express his true love for his better half, Mrs. Sukanya Basu Sarkar, who has always been a pillar of strength and inspiration to the author, and who probably is also one of the true and passionate critic and admirer of the author's work.

The author sincerely wishes his gratitude and love for the organization he is an extremely proud employee of, BMC Software India Pvt Ltd. I LOVE YOU, BMC!!!

And, finally, the author would like to express his humble and sincere gratitude to IBM Corporation, whose work on Quantum Computing and uncountable other technologies, has truly inspired the author to study and research on the field of Quantum Computing.

\section{REFERENCE}

[1] Abramsky S.; Duncan R. (2006). A categorical quantum logic - Mathematical Structures in Computer Science, Volume 16, Issue 3; Cambridge University Press, (pp.469 - 489).

[2] Acín A. (2001). Statistical distinguishability between unitary operations - Physical Review Letters, 87, art. no. 177901; American Physical Society, (https://doi.org/10.1103/PhysRevLett.87.177901).

[3] Aerts D.; Czachor M. (2004). Quantum aspects of semantic analysis and symbolic artificial intelligence, Volume 37, Number 12; Journal of Physics A: Mathematical and General, (pp. L123-L132).

[4] Aïmeur E.; Brassard G.; Gambs S. (2006). Machine learning in a quantum world, vol. 4013; Conference of the Canadian Society for Computational Studies of Intelligence - Proceedings of Canadian AI 2006, (pp. 431442).

[5] Altenkirch T.; Grattage J. (2005). A functional quantum programming language; Proceedings of the 20th Annual IEEE Symposium on Logic in Computer Science (LICS), (pp. 249-258).
[6] Andresen S.L. (2002). John McCarthy: Father of AI; IEEE Intelligent Systems, (pp. 84-85).

[7] Baltag A.; Smets S. (2006). LQP: The dynamic logic of quantum information - Mathematical Structures in Computer Science, Volume 16, Issue 3; Cambridge University Press, (pp. 491-525).

[8] Barenco A.; Bennet C.; Cleve R.; DiVincenzo D.P.; Margolus N.; Shor P.; Sleator T.; Smolin J.A.; Weinfurter H. (1995). Elementary gates for quantum computation, Physical Review A 52, 3457; American Physical Society, (pp. 3457-3467).

[9] Benioff P.A. (1980). The computer as a physical system: A microscopic quantum mechanical Hamiltonian model of computers as represented by Turing machines; Journal of Statistical Physics, 22, (pp. 563-591).

[10] Abramsky S. (2004). High-level methods for quantum computation and information; Proceedings of the 19th Annual IEEE Symposium on Logic in Computer Science, (pp. 410-414).

[11] Abramsky S.; Coecke B. (2004). A categorical semantics of quantum protocols; Proceedings of the 19th Annual IEEE Symposium on Logic in Computer Science, (pp. 415-425).

[12] Benjamin S.C.; Hayden P.M. (2001). Multiplayer quantum games, Physical Review A, 64, 030301(R); American Physical Society, (art. no. 030301).

[13] Bennet C.H. (1973). Logical reversibility of computation; IBM Journal of Research and Development, 17, (pp. 525532)

[14] Bernstein E.; Vazirani U. (1997). Quantum complexity theory; SIAM Journal on Computing, 26, (pp. 1411-1473)

[15] Bertoni A.; Mereghetti C.; Palano B. (2003). Quantum computing: 1-way quantum automata; Proceedings of 7th International Conference on Developments in Language Theory, LNCS, vol. 2710, Springer, (pp. 1-20). 